Editorial

\title{
Diet-Therapy in Pediatric Crohn's Disease: A New Approach
}

Azita Hekmatdoost*

Dept. of Clinical Nutrition and Diet Therapy, National Nutrition and Food Technology Research Institute, Faculty of Nutrition Sciences and Food

Technology, Shahid Beheshti University of Medical Sciences, Tehran, Iran

$\mathrm{I}$ nflammatory bowel disease (IBD), including ulcerative colitis (UC) and Crohn's disease (CD), has become one of the most common chronic conditions all over the world. The prevalence of IBD is growing rapidlyin developing countries along with nutrition transition. IBD affects children as well as adults, with $15-20 \%$ of patients being diagnosed during childhood. The onset of disease during childhood is important because it affects child growth and development $(1,2)$. Moreover, when IBD initiates during childhood, it is usually more severe and involves more regions of the gut. Weight loss, failure to thrive, low bone density, and late puberty are the typical problems in pediatric $\mathrm{CD}$ due to malnutrition which subsequently results in malabsorption. Treatment by corticosteroids aggravates growth problems and worsens malnutrition in the affected children. Currently, exclusive enteral nutrition (EEN) is recommended as the first line of treatment in pediatric CD (3). Metaanalysis of clinical trials has shown that EEN is as effective as corticosteroids in giving rise to remission in children suffering from CD, with $80 \%$ success of active $\mathrm{CD}$ treatment (4). If patient does not respond to EEN after 2 weeks, other therapeutic options should be considered. If two weeks administration of EEN induces remission, EEN should be continued for 6-8 weeks. Then, solid foods can be introduced gradually to the patients' diet during a period of 2-3 weeks. The first group of solid foods, which can be consumed, are low fiber grains such as white flour bread, crackers, pasta, and rice. After 3-4 days, low fat meats and alternatives such as plain (not fried, not processed) lamb, veal, beef, chicken, turkey, fish (low fat), tofu, and eggs can be used. From the tenth day, low fiber fruits and vegetables such as fruits without skin/seeds, cooked vegetables without skin/seeds can be eaten. Finally, low fat dairy products are added to the diet on the fifteenth day, and gradually patients can use a regular diet as tolerated. For maintenance, patients can use EEN in one out of every 4 months in the year or be fed nocturnal partial enteral nutrition (PEN) for 4-5 days every week $(3,5)$.

Current evidence has shown that neither type of the formula nor the location of gut injury is a determinant of the effectiveness of EEN for CD treatment in children. If patient prefers to drink formula, then polymeric formulas are good choices for feeding. When the child cannot drink the whole volume of dietary needs, the patient can be fed by naso-gastric (NG) tube (6).

The mechanism of action in EEN treatment of CD has not yet been fully elucidated, however, the probable mechanisms includes low allergen load, absence of food additives, being nucleotide free, and an anti-inflammatory lipid composition. Recently, it has been hypothesized that EEN has a specific effect on the intestinal microbiome, positively interfering with the dysbiosis in CD patients $(3,5,7)$. Further studies are needed to evaluate the exact mechanism of action of this treatment. Moreover, producing high quality formulas with good taste and low price are absolutely needed in low income countries because the cost of long EEN is not affordable for many patients in these countries. 


\section{References}

1. Dabritz J, Gerner P, Enninger A, Classen M, Radke M. Inflammatory Bowel Disease in Childhood and Adolescence. Deutsches Arzteblatt international. 2017;114(19):331-8.

2. Hekmatdoost $\mathrm{A}, \mathrm{Wu} \mathrm{X}$, Morampudi V, Innis SM, Jacobson K. Dietary oils modify the host immune response and colonic tissue damage following Citrobacter rodentium infection in mice. American journal of physiology Gastrointestinal and liver physiology. 2013;304(10):G917-28.

3. Ashton JJ, Gavin J, Beattie RM. Exclusive enteral nutrition in Crohn's disease: Evidence and practicalities. Clin Nutr. 2018 Feb 1. pii: S0261-5614 (18) 30038-4.

4. Swaminath A, Feathers A, Ananthakrishnan AN, Falzon L, Li Ferry S. Systematic review with metaanalysis: enteral nutrition therapy for the induction of remission in paediatric Crohn's disease. Alimentary pharmacology \& therapeutics. 2017;46(7):645-56.

5. Assa A, Shamir R. Exclusive enteral nutrition for inducing remission in inflammatory bowel disease in paediatric patients. Current opinion in clinical nutrition and metabolic care. 2017;20(5):384-9.

6. Cohen-Dolev N, Sladek M, Hussey S, Turner D, Veres $\mathrm{G}$, Koletzko S, et al. Differences in outcomes over time with exclusive enteral nutrition compared to steroids in children with mild to moderate Crohn's Disease: results from the GROWTH CD study. J Crohns Colitis. 2018 Feb 28;12(3):306-312

7. Connors J, Basseri S, Grant A, Giffin N, Mahdi G, Noble A, et al. Exclusive Enteral Nutrition Therapy in Paediatric Crohn's Disease Results in Long-term Avoidance of Corticosteroids: Results of a Propensityscore Matched Cohort Analysis. Journal of Crohn's \& colitis. 2017;11(9):1063-70. 